\title{
Reply to Nuovo et al.
}

\author{
Hanno J. Bolz ${ }^{1,2}$
}

Received: 24 April 2018 / Accepted: 24 April 2018 / Published online: 25 May 2018

(c) European Society of Human Genetics 2018

Nuovo et al. [1] report on an additional patient with severe congenital ataxia and intellectual impairment whose disease is obviously due to a heterozygous de novo SPTBN2 mutation, p.R480W. The SPTBN2 genotype-phenotype correlation proposed by us, with biallelic loss-of-function mutations causing severe congenital ataxia with cognitive impairment-in contrast to in-frame (mostly missense) events in the gene leading to a milder, dominantly inherited form of ataxia (SCA5) - likely does apply as a general rule/ in most cases.

Here, Nuovo et al. propose that sheer heterozygosity for p.R480 $\mathrm{W}_{S P T B N 2}$ may be sufficient to cause the (for a heterozygous allele unexpected) SCAR14-like phenotype. They review previous descriptions of patients with p.R480W and point out their concordant very severe phenotype. According to in silico analysis described now, and in contrast to other SPTBN2 missense mutations which cause SCA5, p.R480W appears to increase protein stability. Together with the de novo occurrence of p.R480W in different patients, this may indicate that the severe phenotype associated with this particular missense mutation manifests independently of hidden SPTBN2 mutations in trans or modifiers elsewhere. The letter by Nuovo et al. is an example that revisiting known variants is important. p.R480W may not be the only SPTBN2 missense variant causing a phenotype resembling the allelic recessive disorder, SCAR14. Consequently, any rare heterozygous SPTBN2 missense variant in congenital severe ataxia with intellectual disability deserves particular attention. The case of p.R480W demonstrates that also in diagnostic settings, examination of such variants beyond routine procedures may be necessary in order to reach the best conclusion.

\section{References}

1. Nuovo, S. et al. Between SCA5 and SCAR14: delineation of the SPTBN2 p.R480W-associated phenotype. Eur J Hum Genet. 2018;26:928-29.

Hanno J. Bolz

h.bolz@senckenberg-humangenetik.de

1 Senckenberg Centre for Human Genetics, Frankfurt am Main, Germany

2 Institute of Human Genetics, University Hospital of Cologne, Cologne, Germany 